\title{
Numerical modelling and passive flow control using porous media
}

\author{
Charles-Henri Bruneau and Iraj Mortazavi \\ Université de Bordeaux, INRIA Team MC2, CNRS UMR 5466 \\ 351 cours de la Libération, F-33405 Talence \\ bruneau@math.u-bordeaux.fr, mortaz@math.u-bordeaux.fr
}

\begin{abstract}
The whole flow over a solid body covered by a porous layer is presented. The three main models used in the literature to compute efficiently the fluid flow are given: the reduction of the porous layer to a boundary condition, the coupling of Darcy equation with Navier-Stokes equations and the Brinkman-Navier-Stokes equations or the penalisation method. Numerical simulations on Cartesian grids using the latest model give easily accurate solutions of the flow around solid bodies with or without porous layers. Adding appropriate porous devices to the solid bodies, an efficient passive control of the two-dimensional incompressible flow is achieved. A strong regularisation of the flow is observed and a significant reduction of the vortex induced vibrations or the drag coefficient is obtained.
\end{abstract}

Key words : incompressible bluff body flows, passive control, porous media, penalisation method

\section{Introduction}

The aim of this paper is to control the two-dimensional incompressible flow around a solid body by adding porous layers on the surface to change the shear forces in order to regularise the flow and to reduce the vortex induced vibrations or the drag coefficient. Modelling the physics of three different solid, porous and fluid media is an important topic in engineering problems. It needs to describe correctly the boundary conditions between the three regions and in some cases to solve the equations corresponding to the flow inside the fluid and the porous medium $[34,7,31]$. This is a priori difficult to handle as the governing equations are different and the coupling at the interface is not straightforward [27]. A review of the various models developped these last decades is presented in order to select the most appropriate model for the high porosity porous medium and the complex geometries considered in this work.

In the literature several approaches are proposed to study this problem. If the goal is to solve the fluid flow with a porous interface, one can avoid to solve the porous flow imposing correct porous-fluid boundary conditions [5]. This approach is widely used to study turbulent flows over permeable walls for instance [20, 23]. However some authors think that it is necessary to compute the flow in both regions to have a good representation of the porous flow $[21,31,7]$. Then a coupling between Stokes or Navier-Stokes equations and Darcy equations is required with a right treatment of the interface. In our opinion, it is easier to solve this problem by a unique model, namely Brinkman-Navier-Stokes equations or the penalisation method. This consist in adding a term $U / K$ into the Navier-Stokes 
equations where $K$ is a non dimensional permeability coefficient representing the medium. This method can be seen as a fictitious domain method which is very easy to implement, robust and efficient. It does need neither to have a mesh fitting the obstacle nor to impose a boundary condition at the boundary of the solid or an interface condition between the porous and the fluid media $[1,14,6,35]$. Let us note that Brinkman equation is valid only when the porosity of the porous medium is close to one.

The main advantages of passive control is that it is energy free and often easier to implement. Many devices have been proposed in the literature, let us mention compliant walls like the dolphin skin $[38,17]$, ribelets or bumps $[18,25,3]$, splitter plates [2], wavy or rough surfaces $[40,39]$. An other possibility is to introduce porous or permeable layers [9]. The main effect of a porous interface between the solid and the fluid is to change the shear forces. Indeed, the no-slip boundary condition is changed to a quasi slip Fourier type boundary condition due to the Darcy flow inside the porous layer and so the rate of vorticity generation is reduced. Therefore the shedding around a bluff body is modified and the flow behaviour can be drastically regularised as we shall see below. The efficiency of this passive control is related to the choice of the permeability of the porous medium, the thickness of the porous layers and their location. So the setup can be different when the goal is to reduce the vortex induced vibrations of a riser pipe or the drag coefficient of a ground vehicle. This work is only devoted to two-dimensional numerical simulations; it appears that two-dimensional studies are relevant for the flow around a riser pipe $[4,26]$ or a square back Ahmed body [19] as the flow can be a two-dimensional based flow. In any cases a qualitative effect of the porous interface can be obtained as many numerical tests are affordable.

This paper is organised in two main sections: The solid-porous-fluid models including the physical description, the reduction of the porous layer to a boundary condition, the coupling of Darcy or Brinkman equations with Stokes or Navier-Stokes equations and the penalisation method. The numerical simulation and passive flow control including the numerical simulation, the passive control setup and flow regularisation, the control of vortex induced vibrations around a riser pipe section and the control of the drag coefficient of ground vehicles.

\section{The solid-porous-fluid models}

\subsection{The physical description}

In this section the physical properties of a fluid-porous-solid configuration with a high porosity ( $\Phi$ close to 1$)$ medium are described. Indeed, for the passive control a high porosity material is needed [9]. Modelling such phenomena needs to understand correctly the flow behaviour in the porous media, especially in the vicinity of the solid and fluid boundaries. It is also necessary to model correctly the fluid flow in the boundary layer close to the porous interface. In total there are five different regions of the flow from the solid body to the open flow in the fluid as illustrated on Figure 1 where for the sake of simplicity the velocity $U=(u, v)$ is assumed to be parallel to the wall $(v=0)$ :

- the boundary layer in the porous medium close to the solid wall, 
- the homogeneous porous flow with Darcy velocity,

- the porous interface region with the fluid,

- the boundary layer in the fluid close to the porous frontier,

- the main fluid flow.

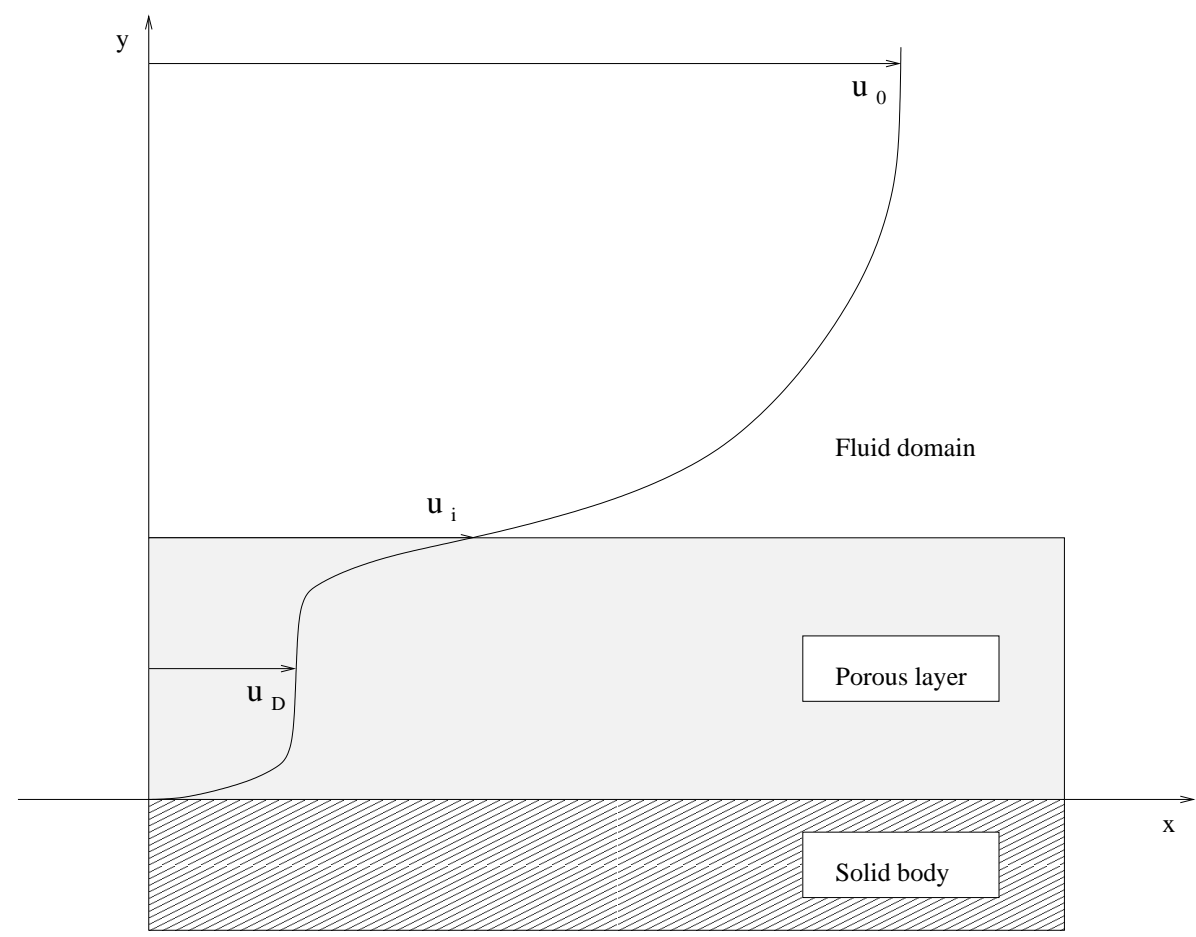

Figure 1: Velocity profile in the vicinity of the porous layer.

According to [34], the effects of a solid boundary to a porous medium flow defers from the conventional fluid-solid boundary layer as the frictional effects are different. As the convective velocity $u_{D}$ is very low, the boundary-layer growth is significant only over a short length $k u_{D} / \nu_{p}$ (where $k$ is the intrinsic permeability of the porous medium and $\nu_{p}=\mu_{p} / \rho$ is the effective kinematic viscosity of the medium with $\mu_{p}$ the effective dynamic viscosity and $\rho$ the density of the medium) and the boundary layer thickness is of order $k^{1 / 2}$ for a porosity close to one. This part of the porous flow is not really significant as there is a Darcy velocity $u_{D}$ in the main region of the porous layer which is essential for the interface with the fluid flow [37]. This Darcy velocity is the major ingredient of the passive control as we shall see in the next section.

The interface between the porous and the fluid regions is the most complex part of this flow. It was the subject of many researches and we can find a lot of papers in the literature (see for instance [28] and [31] and the references therein). There are two parts, an interface inside the porous region and a pseudo boundary layer inside the fluid region. It is shown in the papers above and in [7] that the porous interface thickness scale is about $k^{1 / 2}$ and that the velocity evolves from the Darcy velocity $u_{D}$ to the interface velocity $u_{i}$ with a 
growth of order $k^{1 / 2}$. Therefore, the whole porous layer is divided in three parts. Two parts are very thin and correspond to the neighbourhood of the solid wall and the interface with the fluid. Nevertheless, the main part of this porous layer is an homogeneous porous flow with a Darcy velocity (Figure 1).

In the fluid region a boundary layer develops from the interface velocity $u_{i}$ instead of zero for the conventional one over a solid wall. This layer in the fluid respects the Prandtl boundary layer theory and the only difference with the solid case is the existence of a non zero velocity. It means that the boundary layer growth is determined by the velocity $u_{0}-u_{i}$ where $u_{0}$ is the upstream uniform velocity in the fluid. That means that $\frac{\delta}{x}$ is proportional to $\frac{\left(u_{0}-u_{i}\right) x}{\nu}$ where $\delta$ is the boundary layer thickness, $x$ is the distance from the origin and $\nu$ is the kinematic viscosity of the fluid. Beyond this boundary layer the fluid motion is governed by the usual Navier-Stokes equations.

To reproduce the above physical behaviour several approaches are used. When the study focuses only on the fluid flow, it is possible to solve the Navier-Stokes equations in the fluid with a convenient boundary condition that approximates the interface velocity $u_{i}$. Otherwise, it is necessary to solve the flow in both the porous and the fluid regions. To achieve this goal, two models are commonly used. The three models are presented in the next three sections.

\subsection{The reduction of the porous layer to a boundary condition}

The first approach is based on the pioneer work of Beavers and Joseph [5] in a channel with one permeable wall. Starting from the one-dimensional Darcy law

$$
u_{D}=-\frac{k}{\mu_{p}} \frac{\partial p}{\partial x}
$$

where $p$ stands for the pressure, they postulate that in the porous interface the flow velocity changes rapidly from $u_{D}$ to the slip velocity at the interface $u_{i}$. Assuming that the slip velocity for the free fluid is proportional to the shear rate at the permeable boundary, they relate the flow velocity to the interface velocity by the ad hoc boundary condition

$$
\frac{\partial u}{\partial y}=\frac{\alpha}{k^{1 / 2}}\left(u_{i}-u_{D}\right) ; v=0
$$

where $\alpha$ is the slip coefficient depending on the characteristics of the porous medium. Then, integrating this equation in the channel with no-slip boundary condition on the solid wall, they get the velocity profile and deduce the value of $u_{i}$ as a function of $\partial p / \partial x$. Let us note that this boundary relation was verified by laboratory experiments but can not be used directly for numerical simulations as $u_{i}$ is unknown. Many authors were inspired by this idea to derive numerical boundary conditions on permeable walls. For instance, Hahn et al. [20] rewrite the condition as

$$
\frac{\partial u}{\partial y}=\frac{\alpha}{k^{1 / 2}}\left(u-u_{D}\right) ; v=0
$$

and apply it to compute the turbulent flow in a channel with two permeable walls. Stating that the slip phenomenon is essentially due to the shear stress Jones [24] proposed a 
modification of Beavers and Joseph condition

$$
\left(\frac{\partial v}{\partial x}+\frac{\partial u}{\partial y}\right)=\frac{\alpha}{k^{1 / 2}}\left(u_{i}-u_{D}\right) ; v=0
$$

that can be used in the same way.

Another approach consists in considering that the exchange through the porous or permeable wall take place by normal transpiration. That means that the slip velocity is zero and the normal velocity is prescribed as [30]

$$
u=0 ; \frac{\partial v}{\partial y}=0 .
$$

These boundary conditions are commonly used as above [36] or are rewritten as [23]

$$
u=0 ; v=-\beta p^{\prime}
$$

where $\beta$ is the porosity coefficient and $p^{\prime}=p-G(t) x$ is the instantaneous fluctuation of the wall pressure with respect to the mean pressure gradient. An other form of such boundary conditions is proposed in [15] and a mathematical analysis of a similar conditions is performed in [22].

In some cases it is necessary to compute the flow inside the porous domain and so this approach is not satisfactory. The next sections show how to deal with both fluid and porous flows.

\subsection{The coupling of Darcy or Brinkman equations with Stokes or Navier-Stokes equations}

Here we want to model both the flow in the porous layer and in the fluid region. As the fluid is assumed incompressible, the continuity equation

$$
\operatorname{div} U=0
$$

is imposed in the whole domain. This equation must be coupled to the right fluid motion equation. In many applications the Darcy equation

$$
\frac{\mu_{p}}{k} U+\nabla p=0
$$

is used to model the flow inside a porous medium. On the other hand, according to the flow regime studied, the flow in the free region can be modelled either by the Stokes equation

$$
\partial_{t} U-\nu \Delta U+\nabla p=0
$$

for laminar flows or by the Navier-Stokes equation

$$
\partial_{t} U+(U \cdot \nabla) U-\nu \Delta U+\nabla p=0
$$

for higher flow regimes. This is widely used when the flow in both media must be computed and seems direct and easy to handle. However it is necessary to find out the right 
treatment of the interface between the two media as a boundary condition at the interface is needed to solve the Stokes or Navier-Stokes equations. One choice is to impose directly Darcy equation as a boundary condition for the fluid domain at the interface. In [21] a detail of the implementation is given with a finite elements approximation. A second choice is to use one of the boundary conditions proposed in the previous section. For instance some authors use the Jones condition [31, 16].

Another approach is to use Brinkman equation

$$
\frac{\mu_{p}}{k} U-\tilde{\mu} \Delta U+\nabla p=0
$$

where $\tilde{\mu}$ is the Brinkman effective viscosity, either to model the flow in a large porosity region or to represent only the interface with the fluid that can be always considered as a region with a porosity close to one $[28,29,32]$. The main advantage of this approach is that similar equations are prescribed on both sides of the interface. A first choice [28] is to assume that the velocity is continuous and to impose a stress jump at the interface

$$
\mu_{p}\left(\frac{\partial u_{i}}{\partial y}\right)_{\text {porous }}-\mu\left(\frac{\partial u_{i}}{\partial y}\right)_{\text {fluid }}=\frac{\gamma}{k^{1 / 2}} u_{i}
$$

where $\gamma$ is a dimensionless coefficient of order one. This jump condition is derived in order to connect Darcy equation to Stokes equation using the Brinkman correction. A modified version of this condition is given in [7]. A second choice is to apply the Beavers and Joseph boundary condition to the Brinkman Navier-Stokes interface [33].

We have seen in this section how to couple the models chosen in the fluid and in the porous medium regions. It is necessary to have a careful treatment of the condition at the interface between the two and so to have a good representation of the interface as in the previous section.

\subsection{The penalisation method}

As we have seen in the previous section, it is possible to set similar equations in both regions taking Brinkman model in the porous medium. This is valid only when the porosity $\Phi$ of the porous medium is close to one. Starting from the Forchheiner-NavierStokes equations

$$
\rho \partial_{t} U+\rho(U \cdot \nabla) U+\nabla p=-\frac{\mu}{k} \Phi U+\tilde{\mu} \Phi \Delta U
$$

where $\rho$ is the density of the fluid, we have shown in [9] that we get the Brinkman-NavierStokes or penalised Navier-Stokes equations that are valid in the fluid, the porous and the solid regions

$$
\partial_{t} U+(U \cdot \nabla) U-\frac{1}{R e} \Delta U+\frac{U}{K}+\nabla p=0
$$

where $K=\frac{k \Phi \bar{U}}{\nu H}$ is the non dimensional coefficient of permeability of the medium, $R e=\frac{\bar{U} H}{\nu}$ is the Reynolds number based on the mean velocity $\bar{U}$ and the height of the domain $H$. These equations can be specified also in the solid region as shown in [1] and [14]. In the fluid region the permeability coefficient goes to infinity, the penalisation term vanishes 
and we recover the non dimensional Navier-Stokes equations. In the solid region the permeability coefficient goes to zero and it is then equivalent to solve Darcy equation [1]. For numerical applications we set respectively $K=10^{16}$ and $K=10^{-8}$ in the two regions. When the thickness of the porous layer between the fluid and the solid goes to zero, it is shown in [14] that it is equivalent to solve Navier-Stokes equations in the fluid with a Robin boundary condition instead of the usual no-slip one. That gives a mathematical relevance of the Beavers and Joseph type boundary conditions seen above.

In the literature the same Brinkman Navier-Stokes equations can be found with $\frac{U}{R e K^{\prime}}$ or $\frac{U}{R e D a K^{\prime \prime}}$ instead of $\frac{U}{K}$ where $D a$ is the non dimensional Darcy number. In any cases there is a penalisation term with a non dimensional coefficient, in the present work the coefficient $K$ is taken in the range $10^{-3}<K<10$. A parametric study has shown (see [9]) that an optimal value of this parameter for the passive control is $K=10^{-1}$ as we shall see in the next sections. When the fluid is water and $\bar{U}, H$ are of order one, this value corresponds to a porous medium of intrinsic permeability $k$ of order $10^{-7}$ in addition to a porosity close to one. In $[6,35]$ the authors use the penalisation term $\frac{U}{R e K^{\prime}}$ with $K^{\prime}$ in the range $10^{-7}<K^{\prime}<10^{-4}$ to represent a porous medium made of textiles composite materials. That means, as the two permeability coefficients are related to each other by $K^{\prime}=\nu K$, that the porous media considered have similar characteristics.

The main advantage of this formulation is that it is necessary to impose neither a boundary condition nor a coupling procedure at the interface between the fluid and the porous regions. Moreover, it is not necessary to fit the interface with the mesh. This method can be seen also as an immersed boundary or a fictitious domain method. It is possible to compute the flow around a body with or without porous parts embedded on a Cartesian grid. The only task to fulfil is to define the coefficient $K$ on the points where the unknowns are defined. When such a point is inside the fluid, the porous or the solid regions, the value of $K$ is set respectively to $10^{16}, 10^{-1}$ and $10^{-8}$. Then the same equations are solved in the three media.

\section{Numerical simulation and passive flow control}

\subsection{Numerical simulation}

Using the penalisation method, we have to solve in the time interval $(0, T)$

$$
\begin{aligned}
\partial_{t} U+(U \cdot \nabla) U-\frac{1}{R e} \Delta U+\frac{U}{K}+\nabla p & =0 & & \text { in } \Omega_{T}=\Omega \times(0, T) \\
\operatorname{div} U & =0 & & \text { in } \Omega_{T} \\
U(x, 0) & =U_{0}(x) & & \text { in } \Omega \\
U & =G & & \text { on } \Gamma_{0} \times(0, T) \\
\sigma(U, p) n+\frac{1}{2}(U \cdot n)^{-}\left(U-U_{\text {ref }}\right) & =\sigma\left(U_{\text {ref }}, p_{\text {ref }}\right) & & \text { on } \Gamma_{1} \times(0, T)
\end{aligned}
$$

where $\Omega$ is the whole computational domain including the solid and porous regions, $U_{0}(x)$ is the initial datum, $G$ is the Dirichlet boundary condition (for instance $G=(1,0)$ at the entrance section of an open domain) imposed on the part $\Gamma_{0}$ of the boundary $\partial \Omega, \sigma$ is the stress tensor, $n$ is the unit normal vector pointing outside of the domain, $\left(U_{r e f}, p_{r e f}\right)$ is a 
reference flow and with the notation $a=a^{+}-a^{-}$. The last boundary condition is imposed on the artificial frontiers $\Gamma_{1}$ in order to convey the solution without any reflections (see [8] for more details) and avoids the use of buffer zones.

The evolution equations are discretized in time by a second order Gear scheme with an implicit treatment of the linear terms and an explicit treatment of convection term. The primitive unknowns velocity-pressure are set on staggered grids as illustrated on figure(2). The spatial approximation is performed using second-order centred finite differences for

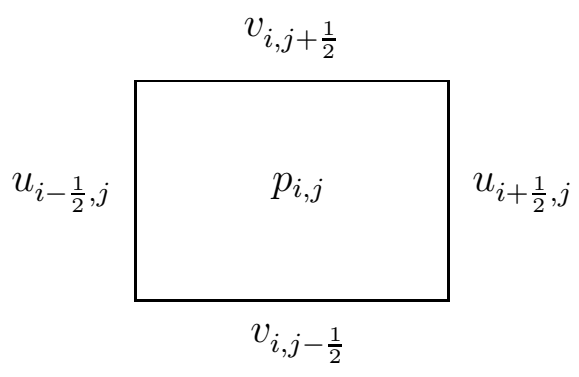

Figure 2: A staggered cell

the linear terms and a third-order upwind scheme for the convection term ([12]). The location of the unknowns enforce the divergence-free equation which is discretized on the pressure points. The whole problem is solved using a multigrid method and on each grid the solution is obtained by means of a cell by cell Gauss-Seidel iterative procedure. As an example, the set of grids varies from the coarsest $25 \times 10$ uniform grid to the finest $3200 \times 1280$ uniform grid to compute the flow around a pipe. The choice of uniform grids is necessary to maintain the accuracy of the finite differences schemes.

\subsection{Passive control setup and flow regularisation}

The aim of this work is to control the flow around a solid body using porous layers on convenient parts of the surface. That means that according to the problem we want to reduce the vortex generation, the vortex induced vibrations (VIV) or the drag forces. These three phenomena are characterised by the enstrophy $Z$, the root mean-square of the lift coefficient $C_{L r m s}$ and the drag coefficient $C_{D}$ defined by

$$
Z=\frac{1}{2} \int_{\Omega}|\omega|^{2} d x ; \quad C_{L r m s}=\sqrt{\frac{1}{T} \int_{0}^{T} C_{L} d t} ; \quad C_{D}=\frac{2 F_{D}}{\rho U_{\infty}^{2} H}
$$

where $\omega$ is the vorticity, $U_{\infty}$ is the upstream flow. The forces are computed thanks to the penalisation method by

$$
F_{D}=\int_{b o d y} \frac{u}{K} d x ; \quad F_{L}=\int_{b o d y} \frac{v}{K} d x
$$

and the lift coefficient $C_{L}$ is defined as $C_{D}$ replacing $F_{D}$ by $F_{L}$. The computation of $F_{D}$ and $F_{L}$ by integration on the body volume is equivalent to the usual computation integrating the pressure and shear forces on the body surface [13]. The $C_{L r m s}$ is directly 
linked to the regularity of the flow and gives a relevant measure of the VIV.

The first numerical test concerns the flow around a rectangular body of size $L=0.2$ in the stream-wise direction and $H=0.16$ in the normal direction. This body is immersed in a computational domain $\Omega=(0,5) \times(0,2)$. The real Reynolds number based on the height of the body is $R e_{H}=300$. The boundary conditions are $U=(1,0)$ at the entrance section $\Gamma_{0}$ on the left and free boundary conditions on the rest of the boundary $\Gamma_{1}$. The passive control is achieved adding a porous layer on the top and on the bottom sides of the rectangle.

We first perform a parametric study on the value of the permeability coefficient $K$ and on the thickness of the porous layer $h[9]$. The two main criteria for this study are the decrease of $Z$ and $C_{L r m s}$. When the value of $K$ is too large $(K>1)$ the flow in the porous medium is close to the fluid flow and the control is not efficient; conversely when $K$ is too small $\left(K<10^{-3}\right)$ the porous medium behaves almost like a solid body. The optimal value is obtained for $K=10^{-1}$. For the thickness of the layer it appears that if the thickness is too low $(h / H<5 \%)$ the darcy flow can not be established inside the porous layer and the control is thus inefficient. In all the simulations we shall take $h / H=10 \%$.

In figure 3 we see that the controlled flow is much more regular than the uncontrolled
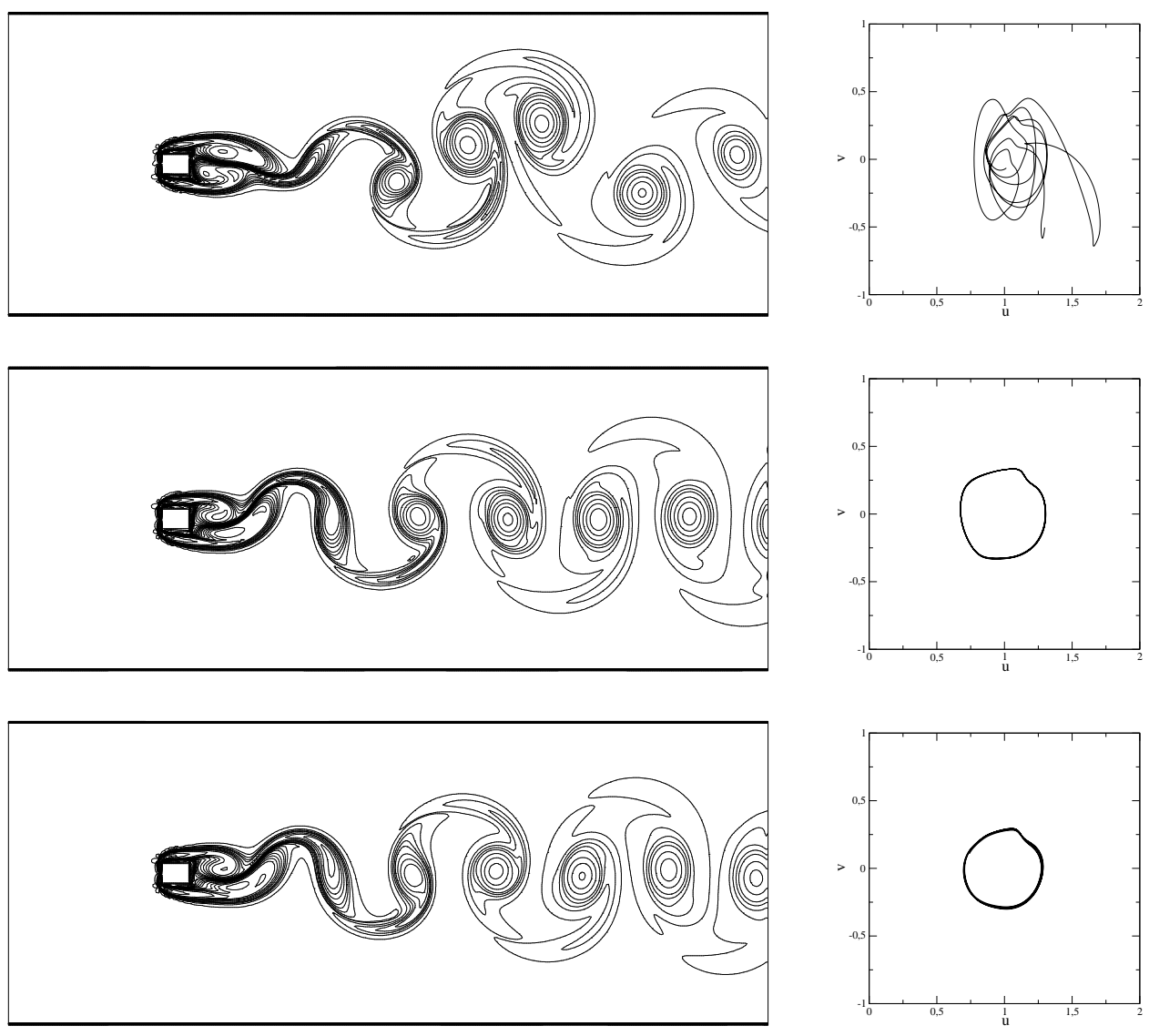

Figure 3: Comparison of vorticity fields and phase portraits at monitoring point $(4.0625,0.75)$ for $R e_{H}=300$ without (top) and with (middle) control in an open domain. The figures at the bottom concern an uncontrolled flow at $R e_{H}=250$. 
one. Indeed, adding the porous layers we recover the Karman street and the solution is really periodic as shown by the phase portrait. To recover such a regular solution without the porous layers, it is necessary to decrease the Reynolds number from $R e_{H}=300$ to $R e_{H}=250$. The regularisation obtained with this passive control is more impressive for higher Reynolds numbers as shown on table 1 as the decrease of $Z$ and $C_{\text {Lrms }}$ becomes larger. This is due to the fact that Darcy flow on both sides of the rectangle stabilises the vortex shedding.

Table 1: Mean value of $Z$ and asymptotic value of $C_{L r m s}$ for various Reynolds numbers.

\begin{tabular}{l|rc|rc|rc}
\hline & \multicolumn{2}{|c|}{$R e_{H}=300$} & \multicolumn{2}{c|}{$R e_{H}=3000$} & \multicolumn{2}{c}{$R e_{H}=30000$} \\
& $Z$ & $C_{\text {Lrms }}$ & $Z$ & $C_{\text {Lrms }}$ & $Z$ & $C_{\text {Lrms }}$ \\
\hline with control & 107 & 0.094 & 410 & 0.221 & 821 & 0.344 \\
without control & 115 & 0.096 & 487 & 0.263 & 1012 & 0.375 \\
\hline
\end{tabular}

\subsection{Control of vortex induced vibrations around a riser pipe section}

In the vicinity of bluff bodies, the shedding of vortices can induce unsteady forces of small amplitude with excitation close to a structural resonant frequency that provoke structural failures. Therefore, the study and the control of vortex shedding has a crucial importance in engineering applications like offshore oil industry. In this case, the VIV can affect the risers. As the environmental conditions are given and can not be changed, the only way to reduce the VIV is to use an efficient control technique adapted to the riser framework. In the present section, we consider a two-dimensional, unsteady and incompressible flow around a fixed circular cylinder of diameter $D=0.16$ immersed in an open computational domain $\Omega=(0,5) \times(0,2)$ with $U=(1,0)$ at the entrance section. This cylinder corresponds to a section of a three-dimensional riser pipe. Such a study with an appropriate choice of the Reynolds number, can give significant informations on the real flow behaviour even if a responding body should be closer to the reality. A parametric study is performed to choose the best $K$ and $h$ values of a uniform porous sheath added around the pipe (figure 4). The tests, for a real Reynolds number $R e_{D}=2400$ based on the di-
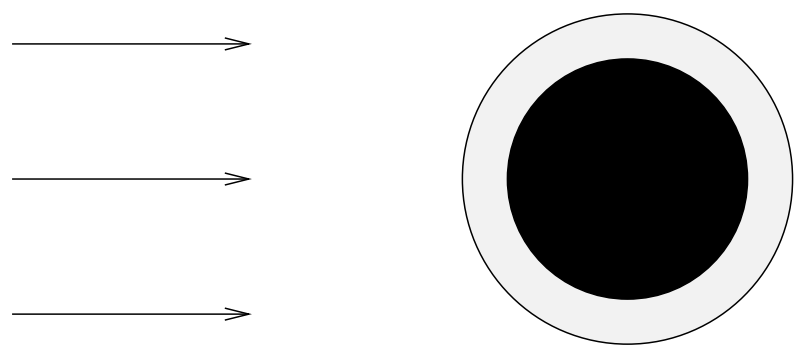

Figure 4: The cylinder with a uniform porous sheath. 
ameter of the cylinder, show again that an efficient control is obtained for $K=10^{-1}$ and $h=0.02$. In addition, it is shown that for such a Reynolds number, the grid convergence is achieved with an uniform grid of $1600 \times 640$ cells. Therefore the results of this section are presented on that grid for the above Reynolds number and on the grid $3200 \times 1280$ for higher Reynolds numbers [10].

The addition of the porous sheath has a tremendous effect on the flow in the vicinity and in the far wake of the pipe. this can be illustrated comparing the velocity signals obtained at monitoring points located behind the cylinder. As an example, these signals are shown on figure 5 at a monitoring point located in the far wake fifteen diameters downstream and one diameter above the symmetry axis. In the uncontrolled case, there are some interactions between the vortices in the wake giving a perturbed signal whereas in the controlled case a pure Karman street is observed yielding a periodic signal with a lower mean value.

A test at a higher Reynolds number $R e_{D}=24000$ is even more impressive as the chaotic

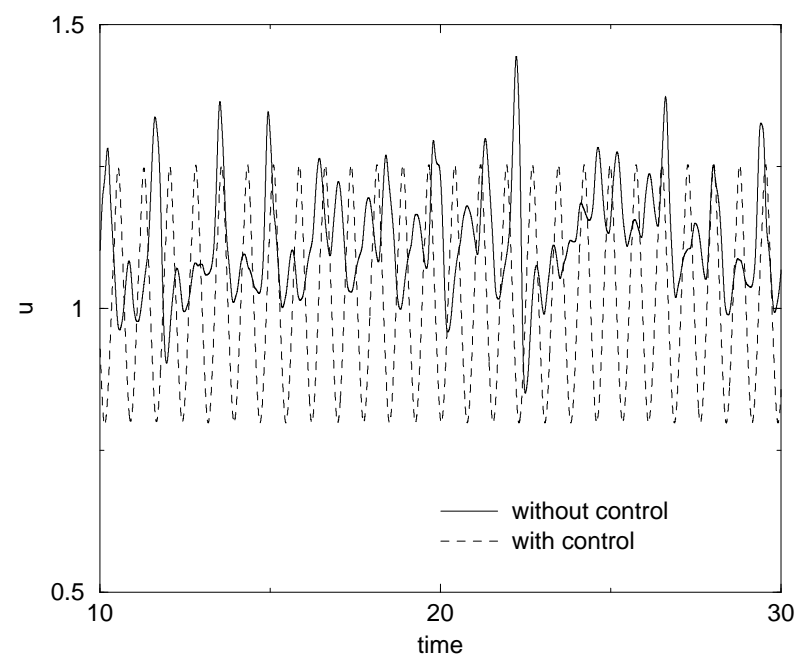

Figure 5: Horizontal velocity histories at monitoring point $(4.0625,0.75)$ at $R e_{D}=2400$.

flow in the wake is regularised towards almost a Karman street (see figure 6). In the first case there are many interactions between the vortices that create more complex dipole or tripole structures advected in all the directions. With the porous sheath, the shear forces are strongly decreased and the quasi slip boundary condition at the porous layer surface induces a much more regular shedding. This is confirmed by plotting the enstrophy history in both cases as on figure 7 . The time function corresponding to the uncontrolled case presents huge variations in the amplitude whereas the function corresponding to the controlled case is almost constant because there is no vortex interactions. Moreover we see on table 2 that there is a tremendous change of the mean value. The regularisation of the flow can be directly read on the values of the $C_{L r m s}$ which is divided by a factor 3.6. This means that the VIV are drastically reduced, therefore the life time of the riser pipe is significantly increased. Finally it appears that the control is more efficient when the Reynolds number increases as can be seen for the two Reynolds numbers considered 


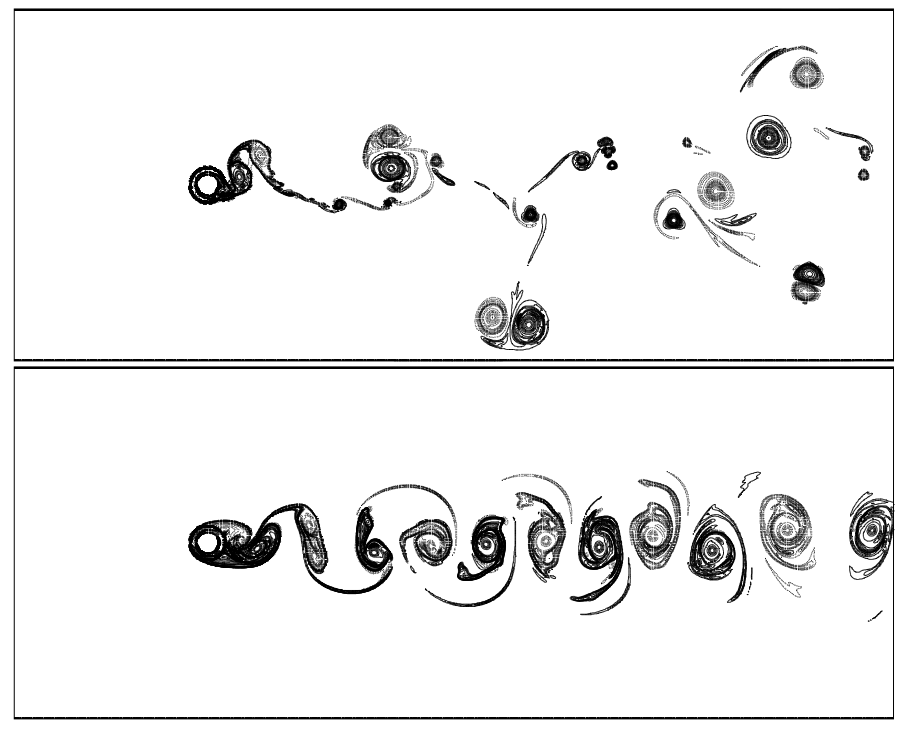

Figure 6: Vorticity field without (top) and with (bottom) control for the same time at $R e_{D}=$ 24000 .

(see table 2).

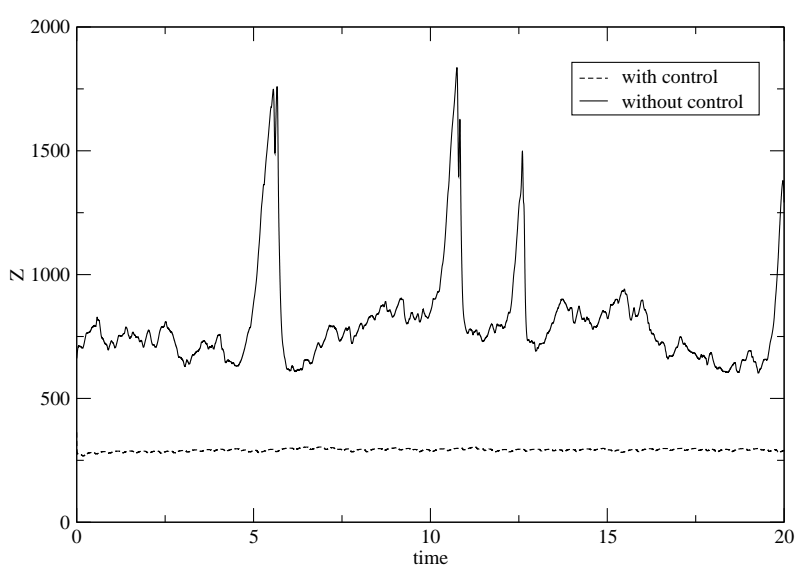

Figure 7: Enstrophy histories at $R e_{D}=24000$.

\subsection{Control of the drag coefficient of ground vehicles}

In this section we want to control the flow around a ground vehicle. This is a very important feature as the goal is to reduce the oil consumption and then the atmospheric pollution. Although it is not yet spread enough, the passive control is very well adapted to car industry as it does not need extra energy. Here the car is represented by a twodimensional cut of the well known Ahmed body and numerical tests are performed for 
Table 2: Mean value of $Z$ and asymptotic value of $C_{L r m s}$.

\begin{tabular}{cc|c|c|c}
\hline$R e_{D}$ & Grid & Simulation & Enstrophy & $C_{\text {Lrms }}$ \\
\hline 2400 & \multirow{2}{*}{$1600 \times 640$} & with control & 190 & 0.125 \\
& & without control & 428 & 0.274 \\
\hline 24000 & \multirow{2}{*}{$3200 \times 1280$} & with control & 291 & 0.081 \\
& & without control & 810 & 0.293 \\
\hline
\end{tabular}

geometries with or without a rear window (see figure 8). Porous devices are included

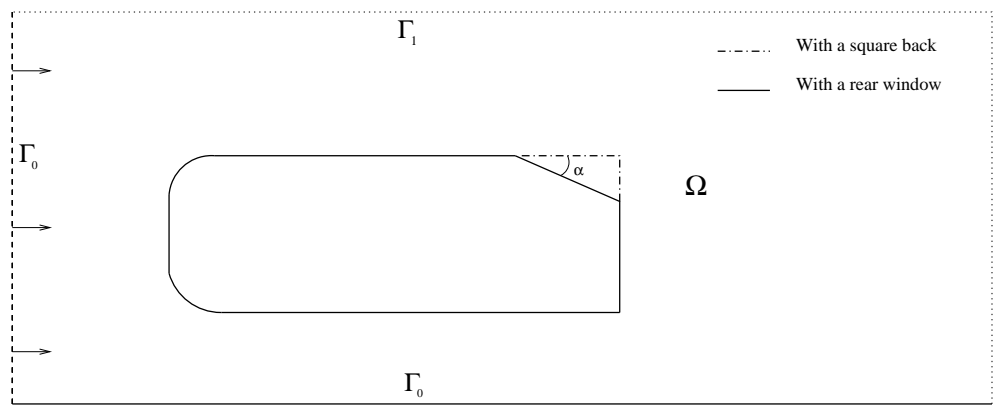

Figure 8: Computational domain including Ahmed body without or with a rear window.

inside the geometry to change the shear forces in order to reduce the drag forces. Indeed, in the car industry the most important aerodynamic parameter is the drag coefficient $C_{D}$. A large number of simulations, with different porous layer locations, were performed to verify how far this passive control technique can contribute to the drag reduction [11]. In the following, only the most significant results are presented starting with the square back geometry. This case corresponds more to a truck for which the flow in the vicinity of the body is mainly two-dimensional. The non dimensional length and height of the Ahmed body are respectively $L=3.625$ and $H=1$. The computational domain is $\Omega=(0,12) \times(0,5)$ with the body located at the distance $d=1$ from the road and the numerical simulations are performed at real Reynolds number $R e_{L}=30000$ on a uniform Cartesian grid of $1536 \times 640$ cells to have a significant number of points in the porous layers of width $h=0.1$. The boundary conditions are of two types, on the entrance section and on the road the constant flow $U=(1,0)$ is imposed and on the two other frontiers the non reflecting boundary condition is used. On figure 9 are shown the two porous devices that give the most interesting results. In the following, the uncontrolled case will be referred as case 0 and the two controlled cases as case 1 and case 2 .

The results are presented on figure 10 where the static pressure coefficient $\left(C_{p}=2(p-\right.$ $\left.p_{0}\right) /\left(\rho|U|^{2}\right)$ with $p_{0}$ the inlet static pressure) isolines are plotted. We see that the pressure gradient in the immediate wake is much lower with the passive control as seen on table 3. This is mainly due to the fact that in the porous layers there is a low speed (less than $10 \%$ of the incoming flow velocity) laminar flow that is expelled at the back in the near wake inducing a weak horizontal jet. The shear forces between the body and the fluid 

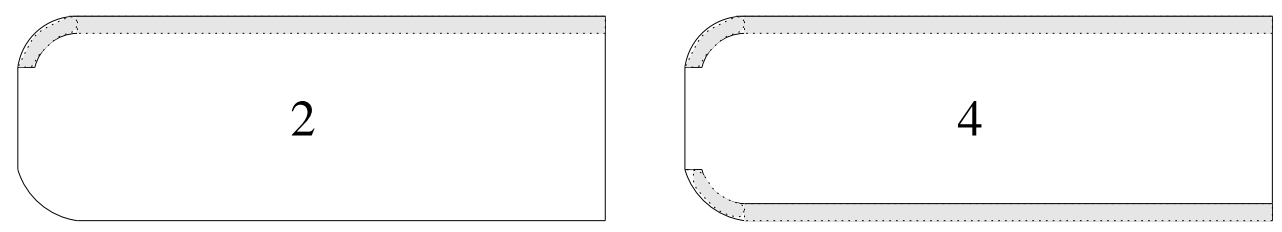

Figure 9: Porous devices for cases 1 (left) and 2 (right) for a square back Ahmed body.

are modified and the horizontal jet at the back decreases the velocity and increases the negative mean pressure significantly (see table 3 ). Consequently the drag at the back (down drag) is drastically reduced as shown on table 4. In case 1 the porous layer on top of the body increases the flow rate in the upper part of the domain and thus decreases the flow rate under the car. So the mean velocity under the car is lower and the aerodynamic power dissipated by the floor of the car is slightly decreased [19] and so the drag of the front part of the body (up drag). In case 2 with porous layers on both sides of the body, the flow rate is the same than in the uncontrolled case. Besides the detached flow is larger at the bottom side of the body, the pressure forces are increased with the resistance to the flow and the up drag is also increased. Consequently, the reduction of the drag coefficient in the second case is weaker despite the very strong reduction at the back. In summary $Z$ and $C_{L r m s}$ are reduced from $23 \%$ to $35 \%$ adding porous layers (case 2 is better than case 1 ) and $C_{D}$ is decreased by more than $30 \%$ (case 1 is better than case 2 ).
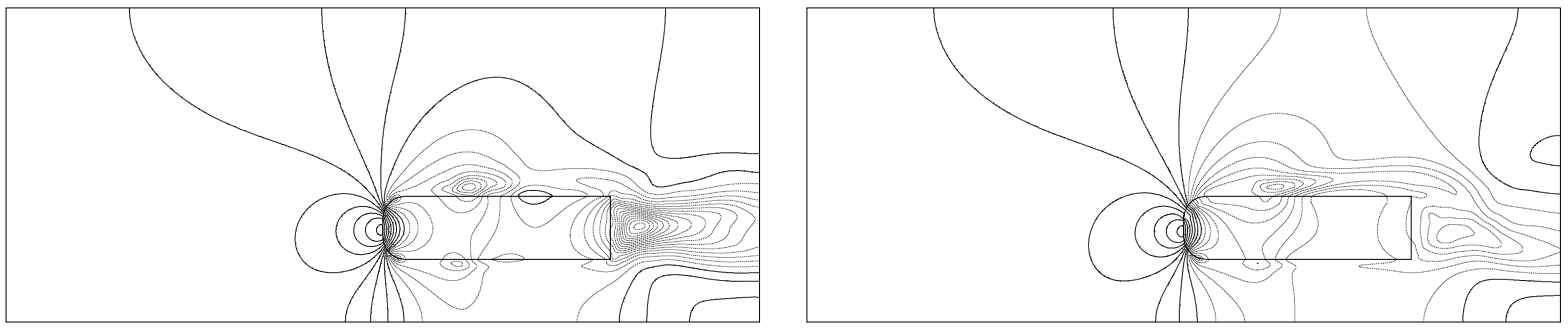

Figure 10: Static pressure coefficient isolines for the flow around the square back Ahmed body on top of a road at $R e_{L}=30000$. Cases 0 (left) and 2 (right).

\begin{tabular}{||l||c|c||}
\hline & $P_{\min }$ value in the wake & $P_{\min }$ location \\
\hline \hline case 0 & -1.636 & $(10.11,1.53)$ \\
case 1 & -0.678 & $(10.22,1.39)$ \\
case 2 & -0.540 & $(10.89,1.34)$ \\
\hline
\end{tabular}

Table 3: The value and the location of the minimum pressure in the close wake of the square back Ahmed body on top of a road at $R e_{L}=30000$.

The second geometry studied is an Ahmed body with an $\alpha=25^{0}$ rear window (see figure 8). For that geometry the flow is not two dimensional any more, there are strong longitudinal vortical structures on both sides of the rear window that interact with the 


\begin{tabular}{||l||c|c|c|c|c||}
\hline & $C_{\text {Lrms }}$ & Enstrophy & Up drag & Down drag & Drag coefficient \\
\hline \hline case 0 & 0.517 & 827 & 0.173 & 0.343 & 0.526 \\
\hline case 1 & $0.396(-23 \%)$ & $592(-28 \%)$ & 0.156 & 0.166 & $0.332(-37 \%)$ \\
\hline case 2 & $0.381(-26 \%)$ & $541(-35 \%)$ & 0.213 & 0.139 & $0.362(-31 \%)$ \\
\hline
\end{tabular}

Table 4: Asymptotic value of $C_{L r m s}$ and mean values of the enstrophy and the drag coefficient for the square back Ahmed body on top of a road at $R e_{L}=30000$.

vortices coming from the shedding at the angle with the roof. However, it is also interesting to see how the shedding is modified by the passive control on the symmetric plane. Of course, a two-dimensional simulation can not give a quantitative measure of the control of the global flow but it permits to get for instance a trend of the evolution of the pressure gradient of the back. With the rear window, the porous devices must stop at the end of the roof and so the effect of the weak horizontal jet, on top of the back, is not beneficial as it increases the size of the recirculation zone on the rear window. It is then necessary to add a porous device on the bottom. But, as we have seen above, the effect on the front is not good, so we modify the porous device to diminish the lower detached zone. The location of the passive control layers are presented, for two different porous devices, on figure 11 .
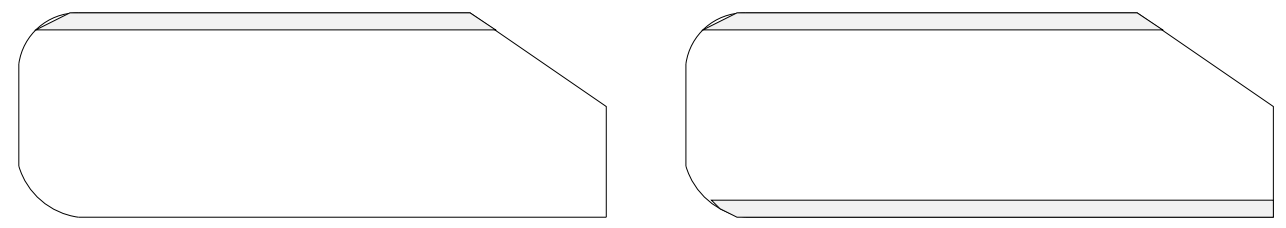

Figure 11: Porous devices for cases 1 (left) and 2 (right) for an Ahmed body with a rear window.
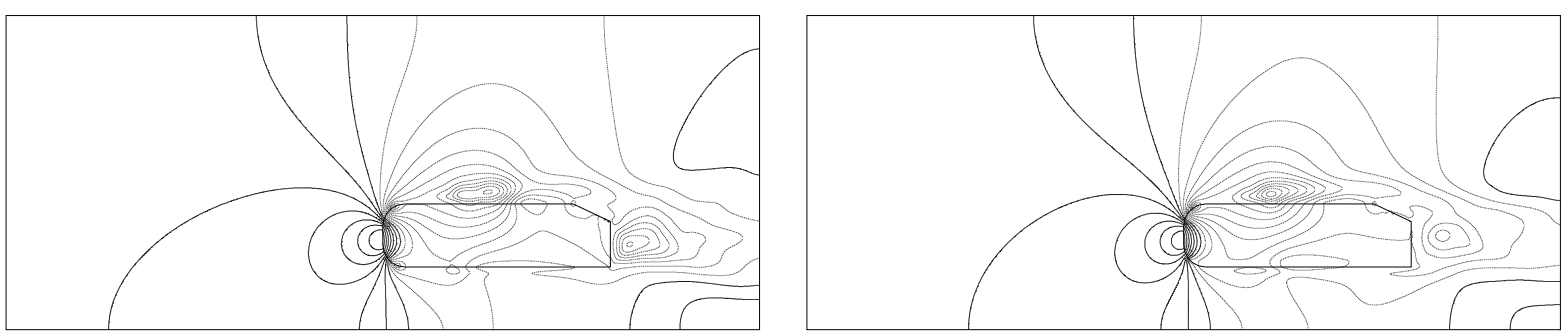

Figure 12: Mean pressure isolines for the flow around Ahmed body with a rear window on top of a road at $R e_{L}=30000$. Cases 0 (left) and 2 (right).

As the table 5 shows, due to the increase of the recirculation zone on the rear window, the minimum of the pressure in the wake $P_{\min }$ for case 1 is lower than for the uncontrolled case. Thus the drag coefficient is higher as shown on table 6. Nevertheless, we observe 
once again that the porous layer has regularised the whole flow as $Z$ and $C_{L r m s}$ are well decreased. To get an improvement of the drag control procedure a porous layer is needed at the bottom. The same effect of the weak horizontal jet as for the square back body is observed at the bottom part in the back. The gradient of pressure is much lower (see figure 12) and thus the drag coefficient (table 6) is decreased as well, as the minimum pressure is increased (table 5). In this case, the main improvement on the drag reduction comes from the down drag, related to the square part of the body. Let us note that if this case is quite efficient for drag reduction, it has no significant regularising effect.

\begin{tabular}{||l||c|c||}
\hline & $C_{p_{\min }}$ value in the wake & $P_{\min }$ location \\
\hline \hline case 0 & -0.813 & $(9.92,1.36)$ \\
case 1 & -1.021 & $(10.06,1.36)$ \\
case 2 & -0.543 & $(10.13,1.50)$ \\
\hline
\end{tabular}

Table 5: The value and the location of the minimum pressure in the close wake of Ahmed body with a rear window on top of a road at $R e_{L}=30000$.

\begin{tabular}{||l||c|c|c|c|c||}
\hline & $C_{\text {Lrms }}$ & Enstrophy & Up drag & Down drag & Drag coefficient \\
\hline \hline case 0 & 0.817 & 726 & 0.099 & 0.176 & 0.282 \\
\hline case 1 & $0.600(-27 \%)$ & $605(-17 \%)$ & 0.100 & 0.190 & $0.300(+6 \%)$ \\
\hline case 2 & $0.801(-2 \%)$ & $670(-8 \%)$ & 0.093 & 0.124 & $0.224(-21 \%)$ \\
\hline
\end{tabular}

Table 6: Asymptotic values of $C_{\text {Lrms }}$ and mean values of the enstrophy and the drag coefficient for Ahmed body with a rear window on top of a road at $R e_{L}=30000$.

\section{Conclusions}

A unique model called the penalisation method is used to simulate the flow inside fluid and porous regions around obstacles. This method is very easy to implement and does not require either a specific treatment of the interface or a body fitting. It is used successfully to introduce a new passive control strategy, which consists in implementing a porous layer between a bluff-body and a fluid, in order to change the boundary layer characteristics. This passive control method yields a drastic regularisation of the flow, especially for high Reynolds numbers. Adding a porous ring around a riser pipe section, the vortex induced vibrations can be devided by more than three. Moreover, with a good choice of the location of the porous layers, the drag coefficient of the square back Ahmed body can be decreased up to $40 \%$.

On the Ahmed body with a rear window, the results are less significant and a mixed strategy coupling active and passive techniques could be beneficial. 


\section{Acknowledgement}

The authors thank warmly their colleagues Didier Lasseux from mechanics department for the fruitful discussions they had on porous media modelling and Patrick Gilliéron from Renault for his interest and collaboration on control of the flow around ground vehicles.

\section{References}

[1] Ph. Angot, Ch.-H. Bruneau and P. Fabrie, A penalization method to take into account obstacles in incompressible viscous flows, Numer Math 199981.

[2] P. W. Bearman, Investigation of the flow behind a two-dimensional model with a blunt trailing edge with splitter plates, J. Fluid Mech. 196521.

[3] P. W. Bearman and M. Branković, Experimental studies of passive control of vortexinduced vibration, Eur. J. Mech. B/Fluids 200423.

[4] P.W. Bearman and J.K. Harvey, Control of circular cylinder flow by the use of dimples, AIAA J. 199331.

[5] G.D. Beavers and D.D. Joseph, Boundary conditions at a naturally permeable wall, $J$. Fluid Mech. 196730.

[6] E.B. Belov, S.V. Lomov, I. Verpoest, T. Peeters and D. Roose, Modelling of permeability of textiles reinforcements: lattice Boltzmann method, Composites Sci. Tech. 200464.

[7] W.P. Breugem, B.J. Boersma and R.E. Uittenbogaard, The laminar boundary layer over a permeable wall, Transport in porous media 200559.

[8] C.-H. Bruneau and P. Fabrie, Effective downstream boundary conditions for incompressible Navier-Stokes equations, Int. J. Num. Meth. Fluids 199419.

[9] C.-H. Bruneau and I. Mortazavi, Passive control of the flow around a square cylinser using porous media, Int. J. Num. Meth. Fluids 200446.

[10] C.-H. Bruneau and I. Mortazavi, Control of vortex shedding around a pipe section using a porous sheath, Int. J. Offshore Polar Engg. 200616.

[11] Ch.-H. Bruneau, P. Gilliéron, I. Mortazavi, Passive control around the Ahmed body using porous devices, submitted.

[12] C.-H. Bruneau and M. Saad, The 2d lid-driven cavity problem revisited, Computers $\mathcal{E}$ Fluids 200635.

[13] J.-P. Caltagirone, Sur l'interaction fluide-milieu poreux : Application au calcul des efforts exercés sur un obstacle par un fluide visqueux, C. R. Acad. Sci. 1994318 série II.

[14] G. Carbou, Brinkmann model and double penalization method for the flow around a porous thin layer, J. Math. Fluid Mech. 20068. 
[15] P.W. Carpenter and L.J. Porter, Effects of passive porous walls on boundary-layer instability, AIAA J. 200139.

[16] D.B. Das, V. Nassehi and R.J. Wakerman, A finite volume model for the hydrodynamics of combined free and porous flow in sub-surface regions, Adv. Environmental Res. 20027.

[17] C. Davies and P.W. Carpenter, Instabilities in a plane channel flow between compliant walls, J. Fluid Mech. 1997352.

[18] U. Ehrenstein, On the linear stabilities of channel flow over ribelets, Physics of Fluids 1996 8.

[19] P. Gilliéron and F. Chometon, Modelling of stationary three-dimensional detached airflows around an Ahmed Reference Body, ESAIM Proc 19997.

[20] S. Hahn, J. Je and H. Choi, Direct numerical simulation of turbulent channel flow with permeable walls, J. Fluid Mech. 2002450.

[21] N.S. Hanspal, A.N. Waghode, V. Nassehi and R.J. Wakeman, Numerical analysis of coupled Stokes/Darcy flows in industrial filtrations, Transport in Porous Media, 200664.

[22] J. Hlomuka, The linearized non-stationary problem for the permeable boundary NavierStokes flows, Applied Math. Comput. 2004158.

[23] J. Jimenez, M. Uhlmann, A. Pinelli and G. Kawahara, Turbulent shear flow over active and passive porous surfaces, J. Fluid Mech. 2001442.

[24] I.P. Jones, Low Reynolds number flow past a porous spherical shell, Proc. Cambridge Philos. Soc. 197373.

[25] P. Luchini, Asymptotic analysis of laminar boundary-layer flow over finely grooved surfaces, Eur. J. Mech. B/Fluids 199514.

[26] A. Miliou, S.J. Sherwin and J.M.R. Graham, Fluid dynamic loading on curved riser pipes, ASME J. Offshore Mech. Artic Engg. 2003125.

[27] D.A. Nield, A. Bejan, Convection in porous media, Springer, 1999.

[28] J.A. Ochoa-Tapia and S. Whitaker, Momentum transfer at the boundary between a porous medium and a homogeneous fluid, Int. J. Heat Mass Transfer 199538.

[29] B.J. Pangrle, A.N. Alexandrou, A.G. Dixon and D. Dibiasio, An analysis of laminar fluid flow in porous tube and shell systems, Chem. Engg. Sci. 199146.

[30] B. Perot and P. Moin, Shear-free turbulent boundary layers. Part 1. Physical insights into near-wall turbulence, J. Fluid Mech. 1995295.

[31] A.G. Salinger, R. Aris and J.J. Derby, Finite element formulations for large-scale, coupled flows in adjacent porous and open fluid domains, Int. J. Num. Meth. Fluids 199418.

[32] R.A. Silva and M.J.S. de Lemos, Turbulent flow in a channel occupied by a porous layer considering the stress jump at the interface, Int. J. Heat Mass Transfer 200346. 
[33] L.M. de Socio, L. Marino and G. Seminara, Stability and admittance of a channel flow over a permeable interface, Phys. Fluids 200517.

[34] K. Vafai and C.L. Tien, Boundary and inertia effects on flow and heat transfer in porous media, Int. J. Heat Mass Transfer 198124.

[35] B. Verleye, M. Klitz, R. Croce, D. Roose, S. Lomov and I. Verpoest, Computation of the permeability of textiles, Computational textile X. Zeng, Y. Li, D. Ruan and L. Koehl Ed., Springer Verlag, 2006.

[36] C. Wagner and R. Friedrich, On the turbulence structure in solid and permeable pipes, Int. J. Heat Fluid Flow 199819.

[37] S. Whitaker, The method of volume averaging, Kluver, 1999.

[38] O. Wiplier and U. Ehrenstein, Numerical simulation of linear and nonlinear disturbance evolution in a boundary layer with compliant walls, J. Fluids and Structures 200014.

[39] H.Y. Wong, A means of controlling bluff body separation, J. Indust. Aerodynamics 1979 4.

[40] M.M. Zadravkovich, Review and classification of various aerodynamic and hydrodynamic means for suppressing vortex shedding, J. Wind Eng. Indust. Aerodynamics 19817. 